rencontre plus fréquemment dans le lait produit pendant les premiers mois de la lactation.

Cependant Brueckner et Guthrie [118] n'ont pas trouvé de corrélation entre le stade de lactation et la saveur oxydée du lait. King et Dunkley [119] montrent que d'une façon générale la quantité de cuivre que contient naturellement le lait est élevée au début de la lactation et diminue rapidement au cours des deux premiers mois jusqu'à une valeur qui restera sensiblement uniforme durant le reste de la lactation. Cependant ces auteurs ne trouvent pas de corrélation entre le taux élevé de cuivre dans le lait de début de lactation et la sensibilité plus ou moins grande du lait au développement de la saveur oxydée.

5. - Influence de L'État de SANté de LA VAChe lattière

Proks et GroH [41] ont signalé que l'état anormal des organes génitaux des vaches laitières pouvait entraîner la production d'un lait présentant un goût défectueux.

Ces auteurs ont montré que le défaut de goût "huileux-rance» peut apparaître avec une intensité variable d'un quartier à l'autre de la mamelle.

\title{
SUMMARY
}

(A suivre).

This first part, devoted to previous works, finds the authors commenting one after the other : Apparition of taste defects in milk - Conditions of organoleptic examination - Nomenclature of taste defects - The reasons advanced to explain the apparition of taste differences in milk - Influences produced by various agents on the appearance of the defects studied.

\section{TEST POUR LA RECHERCHE DES ANTIBIOTIQUES DANS LE LAIT (1) (Essai sur disque à phase inversée)}

$$
\text { par }
$$

\section{F. V. KOSIKOWSKI et R. A. LEDFORD}

Le besoin d'un test simple d'usage courant pour déceler la pénécilline et les autres antibiotiques dans le lait n'exige pas une grande explication. Ses avantages sautent aux yeux.

(1) Journal of the American Veterinary Association, 1960, 136, na 7, 297. - Le $D^{x}$ Kosikowski est Professeur de Science laitière à l'Université Cornell, Ithaca (N. Y.).

- M. Ledford enseigne la Science laitière dans le même établissement. 
En 1957, a été publiée [3] une étude reposant sur le principe du vide et du gaz pour effectuer des tests de laboratoire standards d'antibiotiques d'utilisation courante. Dans leur forme actuelle, la méthode standard sur disque de l'A.P.H.A [1] et le test de l'administration des produits alimentaires et pharmaceutiques [2] exigent l'ensemencement de gélose nutritive sérum avec des bactéries d'essai avant que le lait à examiner soit appliqué sur les surfaces de gélose par des disques de papier humides. De telles plaques de gélose sérum doivent être conservées à basse température $\left(1,7^{\circ}\right.$ à $4,4^{\circ}$ ) jusqu'au moment de l'examen. L'exposition à la température de la chambre, même pendant une courte période de temps avant l'application du lait, déclenche la croissance des bactéries ensemencées et rend l'essai sans effet.

Des recherches continuelles sur les méthodes prometteuses basées sur le vide et le gaz actuellement à l'étude ont conduit à une nouvelle conception en ce qui concerne le test des antibiotiques dans le lait. Ce nouveau test conçu pour éliminer les restrictions imposées par la croissance rapide des bactéries sur plaques de gélose nutritive donne un résultat en quatre heures ou moins. Ce rapport décrit le principe et les détails de cette conception.

\section{PRINCIPE DE LA MÉTHODE}

Les méthodes d'essai sur disque pour le lait dépendent des spores de Bacillus subtilis comme organisme d'essai. Les spores bactériennes possèdent un avantage sur les cellules végétatives en ce sens qu'aucun transfert antérieur n'est nécessaire pour maintenir une activité complète des organismes d'essai avant l'ensemencement. Une autre caractéristique des spores bactériennes est leur aptitude à survivre presque intactes dans une ambiance défavorable. Ce fait, dont il n'a jamais été tiré parti dans les tests d'antibiotiques dans le lait, est la base de la nouvelle méthode à phase inversée. L'élimination de tous aliments de la couche de gélose déjà ensemencée avec des spores de $B$. subtilis empêche la germination des spores à toute température d'exposition. L'inversion de la phase nutritive et l'incorporation au disque de papier de suffisamment de substances alimentaires permet de déclencher la croissance bactérienne à tout moment simplement en appliquant le disque de papier imprégné de lait sur la surface de la gélose. Ainsi, la mobilité du test à la température de la chambre est possible.

\section{PRODUITS EXIGÉS}

La gélose saline ordinaire a été préparée en dissolvant $30 \mathrm{~g}$ de gélose et $9 \mathrm{~g}$ de chlorure de sodium (CP) avec de l'eau distillée, 
suffisamment pour 1 litre. Cette gélose fut stérilisée à $120^{\circ}$ pendant 15 minutes. Un $p \mathrm{H}$ de 6,3 fut considéré être le plus convenable, ce qui est obtenu sans aucun réglage.

Des suspensions de spores de Bacillus subtilis furent obtenues de Difco Co., Détroit, ou furent préparées dans ce laboratoire.

Des disques nutritifs furent préparés en imprégnant des disques de papier Schleicher et Schuell (no 740-E) dans une solution à 20 p. 100 de peptone et à 20 p. 100 de dextrose et en desséchant par congélation tous les disques en un seul lot.

\section{MÉTHODES}

A des portions de $100 \mathrm{ml}$ de gélose chaude $\left(70^{\circ}\right)$ ordinaire stérile fut ajouté $1 \mathrm{ml}$ de spores de $B$. subtilis; des portions de $10 \mathrm{ml}$ de cette gélose ensemencée furent réparties régulièrement à l'aide d'une pipette sur les surfaces du fond de plaques de Petri en verre ou en plastique de dimension standard. Quand la gélose fut solidifiée, les plaques furent scellées en paquets à l'abri de l'humidité. Ces paquets peuvent être conservés pendant plusieurs semaines à $44^{\circ}$ sans se dessécher.

Pour les essais, la plaque fut retirée du paquet. Un disque spécial en papier chargé d'aliment, imprégné avec le lait à examiner fut appliqué à la surface de la gélose. Les disques furent débarrassés du lait excédentaire par une courte agitation, puis placés à environ $20 \mathrm{~mm}$ de distance sur la surface de la gélose.

Après application du nombre voulu de disques sur la plaque de Pétri, la plaque recouverte fut mise à incubation verticalement à $35-37 \circ$.

De 4 à 6 heures plus tard, les surfaces de gélose, le couvercle étant enlevé, furent examinées à la vue sous la lumière à divers angles. Une surface de croissance près d'un disque indiquait un lait normal, alors qu'une surface claire sans croissance autour du disque, avec ou sans halo, indiquait habituellement la présence d'antibiotiques.

\section{RÉSULTATS}

Sensibilité de la méthode. - Comparée à la méthode standard A.P.H.A. [1], le test d'essai sur disque à phase inversée a une sensibilité égale (Tableau no I). 


\section{TABLEAU I}

EFFET DE LA CONGENTRATION DU DISQUE NUTRITIF SUR LA SENSIBILTÉ DE LA MÉTHODE A PHASE INVERSÉE (I)

\begin{tabular}{|c|c|c|c|}
\hline \multirow{2}{*}{$\begin{array}{c}\text { Disque d'essai } \\
\text { d'un quart de pouce } \\
(6,35 \mathrm{~mm}) \text { desséché avec }\end{array}$} & \multirow{2}{*}{$\begin{array}{c}\text { Croissance } \\
\text { de } B . \text { subtilis } \\
\text { autour du disque } \\
\text { (lait normal) }\end{array}$} & \multicolumn{2}{|c|}{$\begin{array}{c}\text { Diamètre de la zone d'inhibition } \\
\text { (en cm) }\end{array}$} \\
\hline & & $\begin{array}{c}\text { Lait frais } \\
\text { contenant } 0,1 \text { U.I. } \\
\text { de pénicilline } \\
\text { par ml de lait }\end{array}$ & $\begin{array}{c}\text { Lait frais } \\
\text { contenant } 0,5 \text { U.I. } \\
\text { de pénicilline } \\
\text { par ml de lait }\end{array}$ \\
\hline 2,5 p. 100 de peptone. . & $+t$ & 1,3 & - \\
\hline 5,0 p. $100 \ldots \ldots \ldots \ldots$ & +++ & 1,3 & - \\
\hline $10,0 \mathrm{p} .100 \ldots \ldots \ldots \ldots$ & $++t+$ & 1,2 & - \\
\hline 20,0 p. $100 \ldots \ldots \ldots \ldots$ & $+t+t+$ & 1,1 & 1,8 \\
\hline 20 p. 100 de peptone et & & & \\
\hline 20 p. 100 de dextrose.. & $++++t$ & 1,0 & 1,5 \\
\hline
\end{tabular}

(1) Observations après 6 heures à $35^{\circ} .+=$ croissance visible à l'œil. L'augmentation des + indique une plus grande densité de croissance. - = pas de croissance dans l'aire touchant au disque et ni zone ni halo. Ces échantillons sont considérés oomme contenant de la pénicilline.

Des concentrations de pénicilline aussi faibles que 0,05 U.I. de pénicilline par millilitre de lait, furent décelées en utilisant des disques de $6,35 \mathrm{~mm}$. La sensibilité fut supérieure avec de plus grands disques (1) et avec certains produits nutritifs spéciaux sur le disque de papier. Il est raisonnable de s'attendre, lorsque de nouvelles études des disques et des produits nutritifs auront été effectuées à ce que la sensibilité de ce test soit augmentée.

Dans une série d'essais, un disque de papier ordinaire d'un demi pouce $(12,70 \mathrm{~mm})$ de diamètre, imprégné seulement avec du lait contenant 0,1 U.I. de pénicilline par millilitre, donna une zone d'inhibition bactérienne d'un diamètre de 1,3 $\mathrm{cm}$. En utilisant un disque desséché dans une solution de peptone à 20 p. $100 \mathrm{du}$ même lait, on obtint une zone de $1,1 \mathrm{~cm}$; un disque desséché dans une solution de 20 p. 100 de peptone et 20 p. 100 de solution de dextrose, et imprégné avec le même lait donna une zone d'un diamètre de $1,0 \mathrm{~cm}$. La croissance fut la plus lente avec des disques imprégnés

(1) On peut obtenir trois dimensions : $1 / 4$ de pouce $(6,35 \mathrm{~mm}) ; 1 / 3$ de pouce $(8,46 \mathrm{~mm})$ et $1 / 2$ pouce $(12,70 \mathrm{~mm})$. 
seulement de lait. En outre, à des taux de pénicilline de plus de 0,5 U.I. par millilitre, les disques saturés avec du lait seulement ou avec du lait et une solution à $20 \mathrm{p}$. 100 de peptone ne donnèrent aucun effet de halo. Toutefois, il n'y eut aucune croissance dans les surfaces autour des disques, de sorte que de toutes façons le lait aurait été considéré comme contenant de la pénicilline. Avec des disques à 20 p. 100 de peptone, plus 20 p. 100 de dextrose, la croissance fut la plus rapide, la sensibilité fut légèrement réduite et l'effet de halo caractéristique existait avec tout lait contenant des concentrations de pénicilline allant jusqu'à 1,0 U.I.

\section{TABLEAU II}

SENSibIlté de LA MÉthode dU disque d'ESSAI A PHASE INVERSÉ

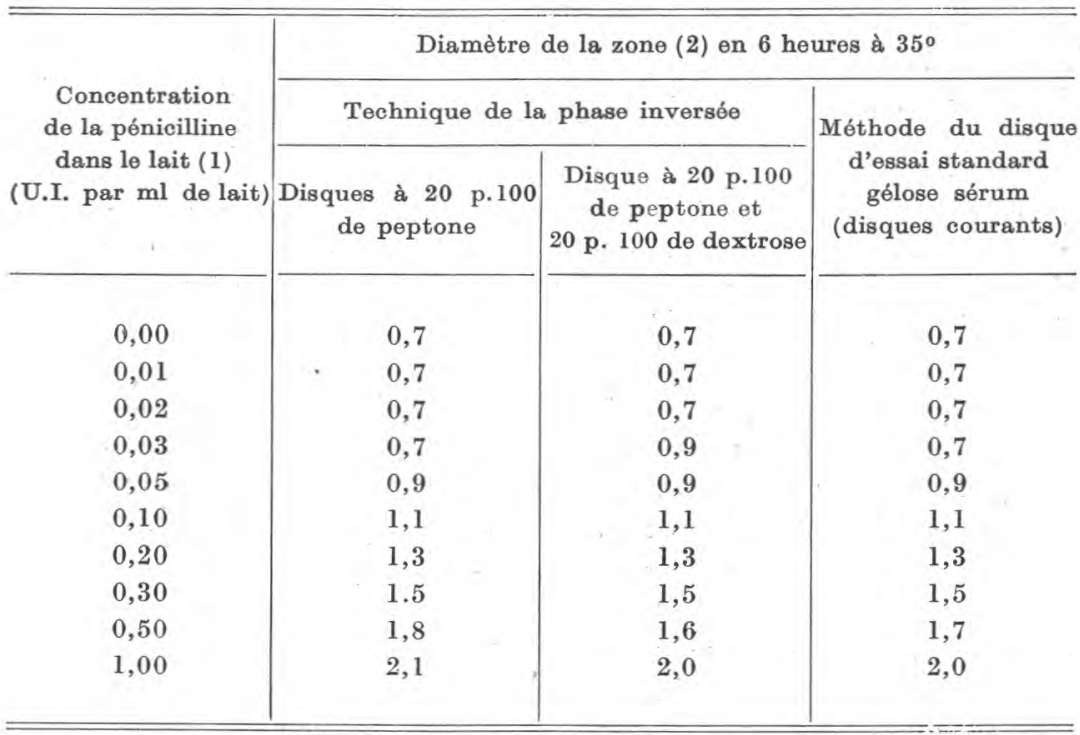

(1) Le lait fut chauffé pendant 2 minutes à $82,2^{\circ}$ après addition de la pénicilline.

(2) Une valeur de $0,7 \mathrm{~cm}$ représente le diamètre du disque lui-même et implique dans cette étude que la zone de croissance au voisinage du disque existait.

D'autres produits nutritifs furent aussi appliqués directement à des disques de papier ordinaire, sous forme pulvérulente. Ceux-ci comprenaient du sérum desséché par pulvérisation (Spray), de la gélose au sérum desséché et de l'l-alanine. Dans tous les essais, les résultats furent moins favorables qu'avec les disques peptone et de dextrose. 


\section{Pour obtenir des résultats en 2 heures $1 / 2$ à 3 heures}

Des résultats sont obtenus bien plus rapidement, si nécessaire, à l'aide d'un microscope utilisant l'objectif à faible puissance. On enlève son couvercle à une plaque après 3 heures d'incubation à 35-37o, et la partie basse de la gélose est retournée sur la platine microscopique. Dans du lait normal exempt d'antibiotique, en utilisant l'objectif à faible puissance, et sans coloration, de très petits filaments comme des colonies sont observés tout près et au voisinage immédiat du disque. Ces petits filaments ne sont pas à confondre avec les petits points ronds noirs qui apparaissent souvent dans le champ de gélose. Si on continue l'incubation, les colonies précoces en forme de filaments prennent une forme arrondie avec des projections aussi minces que des cheveux. Les résultats sont obtenus en un peu plus de deux heures après incubation, à l'aide du microscope ; mais ce sont des cas exceptionnels.

Si la croissance de l'organisme d'essai (B. subtilis) est lente pour quelque raison, y compris l'utilisation de températures d'incubation plus basses que celles recommandées, le décèlement visuel est retardé. Naturellement dans ces conditions, le décèlement microscopique à faible puissance des colonies est aussi retardé.

\section{Durabilité des spores}

Théoriquement les spores bactériennes devraient survivre et ne pas germer en l'absence d'aliments même après exposition à Ia température de la chambre ou à une température plus élevée. Des plaques contenant de la gélose saline ordinaire ensemencées et enveloppées en paquets sous feuilles d'aluminium non perméables, furent conservées pendant 5 jours à $5^{\circ}, 22^{\circ}$ et $35^{\circ}$. A la suite de cette période, du lait normal frais et du lait contenant de la pénicilline furent examinés sur ces plaques. Aux trois températures, la germination des spores en bactéries végétatives n'avait pas eu lieu quand les plaques furent sorties de leurs paquets. Toutefois, quand les disques nutritifs humectés de lait furent appliqués sur les surfaces de gélose, la croissance de $B$. subtilis eut lieu rapidement en 3 heures, quelle qu'ait été la température de conservation. En 6 heures, des halos ou des zones d'inhibition distincts furent observés visuellement sur le lait contenant de la pénicilline (Tableau III). La durabilité de ces spores aux températures indiquées est toujours à l'étude.

\section{Discussion}

La signification des résultats de la présente méthode indique un développement possible d'un test simple avec un degré élevé 
TABLEAU III

EFFET DE TEMPÉRATURES ÉLEVEES DE GONSERVATION SUR L'ESSAI DU DISQUE A PHASE INVERSÉE

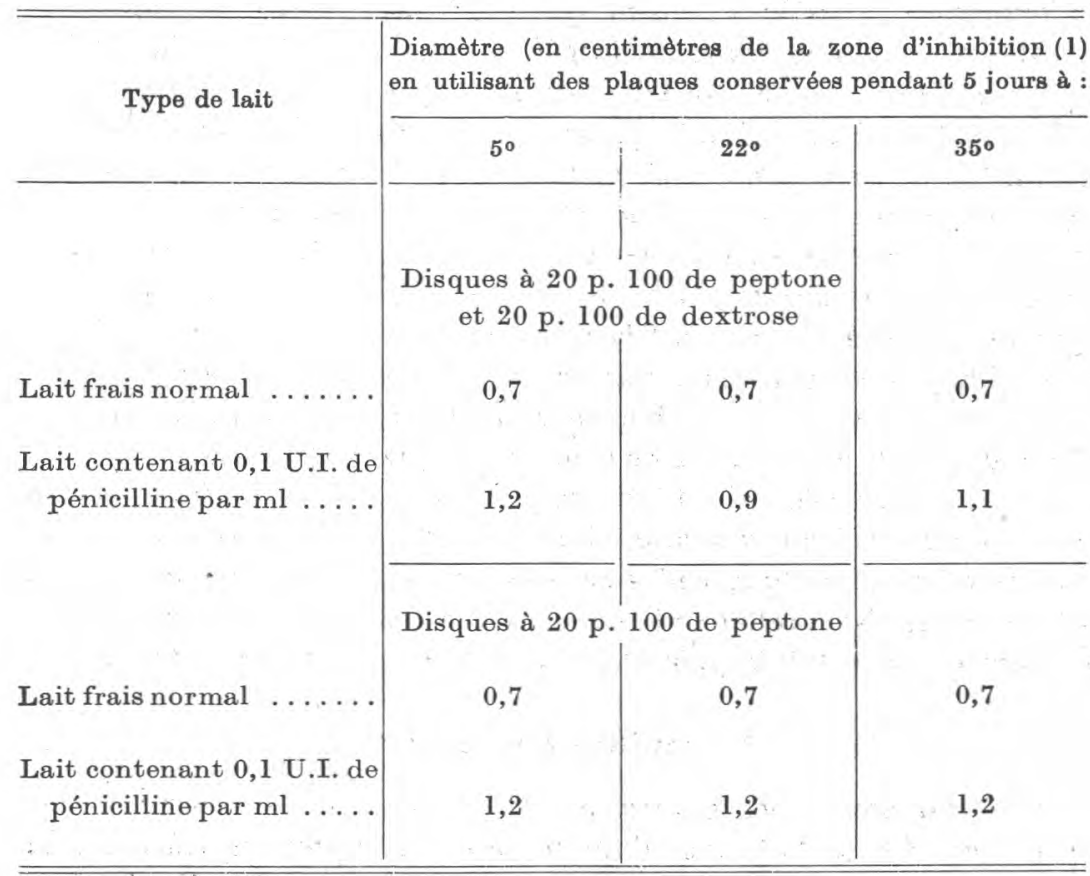

(1) Une valeur de $0,7 \mathrm{~cm}$ représente le diamètre du disque lui-même sans zone. Une bonne croissance près du disque lui-même est obtenue dans tous les cas, lorsque le valeur de 0,7 est indiquée.

de sensibilité et de flexibilité. Pour voir comment il s'adaptera avec d'autres conceptions pour des tests simples pour antibiotiques à l'étude actuellement dans le laboratoire des auteurs, et dont quelques-uns utilisent les principes $d u$ vide et du gaz, il faudra attendre les résultats d'essais pratiques à l'étude actuellement.

Les résultats préliminaires indiquent qu'un test de décèlement simple et peu coûteux des antibiotiques dans le lait sera bientôt à la disposition des vétérinaires, des fonctionnaires de l'hygiène publique, des directeurs de laiterie, et des fermiers, pour être utilisé d'une façon courante. Toutefois, un certain nombre de mesures de précaution sont nécessaires pour exécuter un test courant exact. Elles seront publiées ultérieurement en détail. 


\title{
SUMMARY
}

The principle and details of a new test for detecting antibiotics in milk, called the "reverse-phase disc assay test", are described. This test was designed to provide flexibility in field testing for antibiotics. Storage temperature no longer exerts a great influence on seeded bacteria because nutrients are eliminated from the agar and deposited on test disks instead. Results may be obtained in 6 hours without optical aids and in $21 / 2$ to 3 hours utilizing microscopic examination.

\section{BIBLIOGRAPHIE}

[1] American Public Health Association : Standard Methods for the Examination of Dairy Products. 1953, 10 ${ }^{\text {th }}$ ed., 320-324. (Association Américaine de l'Hygiène Publique : Méthodes normales pour l'exa-

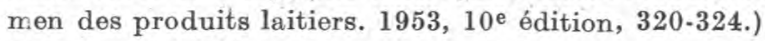

[2] B. Arret and A. Kirshbaum. A rapid disc assay method fort detecting penicillin in milk. J. Milk and Food Tech., 1959, 22, 329-331. (Méthode rapide d'essai avec disque pour déceler la pénicilline dans le lait. J. Milk and Food Tech., 1959, 22, 329-331.)

[3] F. V. Kosikowski. Controlling growth of Test Bacteria for Antibiotic Assays through Anaerobiosis. Science, 1957, 126, 844-845. (Surveillance de la croissance de bactéries de test pour essais d'antibiotiques par anaérobies. Science, 1957, 126, 844-845.)

\section{SUPPLEMENT TECHNIQUE}

\section{L'EMPLOI DE LA CASÉine dANS LES MÉLANGES A BASE DE LATEX SYNTHÉtIQUE POUR LA FABRICATION DU PAPIER COUCHÉ}

\author{
par \\ G. GÉNIN
}

Ingénieur E.P.C.

Depuis quelques années, l'emploi des latex synthétiques que produit l'industrie du caoutchouc, comme liant dans la préparation des mélanges de pigment servant à la fabrication du papier couché, a pris un important développement, et on citera en particulier, parmi ces latex, les latex de styrène-butadiène tels que le Dow Latex512-R fabriqué par la Dow Chemical Co. Ce produit, qui diffère 